\title{
ARTICLE
}

Received 9 Apr 2014 | Accepted 29 Jul 2014 | Published 10 Sep $2014 \quad$ DOl: 10.1038/ncomms5838

\section{Nonlinear phononics using atomically thin membranes}

\author{
Daniel Midtvedt ${ }^{1, \dagger}$, Andreas Isacsson ${ }^{1} \&$ Alexander Croy ${ }^{1, \dagger}$
}

Phononic crystals and acoustic metamaterials are used to tailor phonon and sound propagation properties by utilizing artificial, periodic structures. Analogous to photonic crystals, phononic band gaps can be created, which influence wave propagation and, more generally, allow engineering of the acoustic properties of a system. Beyond that, nonlinear phenomena in periodic structures have been extensively studied in photonic crystals and atomic BoseEinstein condensates in optical lattices. However, creating nonlinear phononic crystals or nonlinear acoustic metamaterials remains challenging and only few examples have been demonstrated. Here, we show that atomically thin and periodically pinned membranes support coupled localized modes with nonlinear dynamics. The proposed system provides a platform for investigating nonlinear phononics.

\footnotetext{
${ }^{1}$ Department of Applied Physics, Chalmers University of Technology, S-41296 Göteborg, Sweden. †Present address: Max Planck Institute for the Physics of Complex Systems, Nothnitzer Str. 38, 01187 Dresden, Germany. Correspondence and requests for materials should be addressed to D.M.

(email: midtvedt@pks.mpg.de) or to A.C. (email: croy@pks.mpg.de).
} 
$\mathrm{N}$ onlinear photonic ${ }^{1}$ and optomechanical crystals $^{2-4}$, as well as atomic Bose-Einstein condensates in optical lattices $^{5-7}$ are well-known examples for which nonlinear phenomena in periodic structures have been intensively studied ${ }^{8}$. All these systems exploit the wide tunability of system properties offered by artificial periodic structures. Similarly, phononic crystals can be engineered to have tailored phonon propagation properties $^{9-11}$. However, nonlinear effects in phononic crystals are, in general, much harder to obtain ${ }^{12}$.

The basic building block of a phononic crystal consists of two coupled localized phonon modes, a phononic dimer. From studies of Bose-Einstein condensates ${ }^{13-15}$ and nanomechanical resonators $^{16-19}$, it is known that the presence of nonlinearities leads to a rich dynamical behaviour even for such a simple system. Moreover, coherent manipulation of phonons in coupled resonators has recently been demonstrated in the linear 'Rabi' regime ${ }^{20,21}$. Accessing the nonlinear regime and the ability to scale the system beyond dimers will be crucial for realizing nonlinear phononics. Nanomechanical systems that exhibit a combination of strong nonlinearities and a high level of control and tunability ${ }^{16-27}$, are promising candidates in this respect.

Here, we show that atomically thin and periodically pinned membranes provide an excellent platform for nonlinear phononics. In particular, they offer high scalability and facilitate the design of arrays of defects. Using a continuum mechanics description $^{28}$ of a pinned graphene membrane, we find that vacancies in the pinning lattice support localized flexural modes, which can be accessed and tuned individually. Two such modes in close proximity interact via the elastic energy and constitute a simple phononic dimer. We show that the system can be tuned from the linear 'Rabi' to the 'Josephson' regime ${ }^{13,15}$. On the basis of the dimer results, our setup can be extended to construct arrays of defects, which support localized modes with adjustable properties. The nonlinear interaction between the localized flexural modes provides a viable path to study phononic manybody effects. Due to the high frequencies of the modes and the low mass of the membrane, our system is an ideal candidate for studies in the quantum regime ${ }^{29}$.

\section{Results}

Pinned graphene membrane. To realize the phononic crystal, we consider a graphene membrane, which is deposited on top of a square lattice of cylindrical pillars with radii $\sigma a$ and lattice vectors $\mathbf{a}_{1}$ and $\mathbf{a}_{2}$ (see Fig. 1). Due to the large adhesion energy, the graphene membrane is essentially pinned at the pillars ${ }^{30,31}$, but free to move otherwise. This situation may be described using a continuum mechanics model ${ }^{28,30}$. The Lagrangian density of a

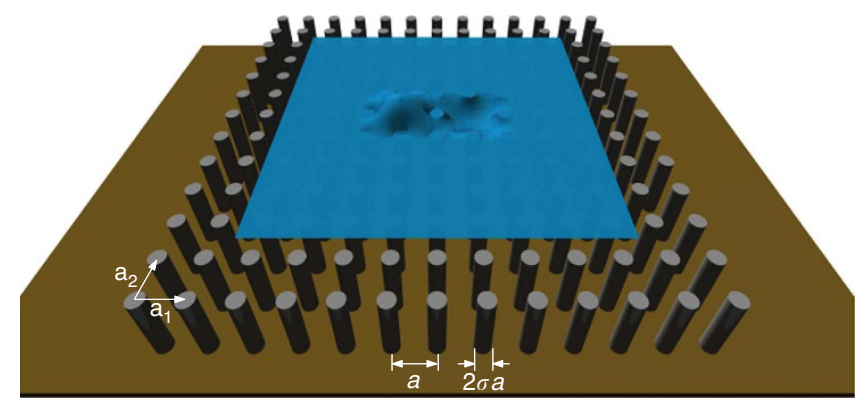

Figure 1 | Schematic illustration of the pinning-lattice setup. Cylindrical pillars with diameter $2 \sigma a$ are arranged in a periodic lattice with lattice spacing $a$. The suspended graphene membrane is pinned at the top of the pillars. By removing pillars, localized modes can be created. graphene sheet subject to a pinning potential $V(\mathbf{r})$ is

$$
\begin{gathered}
\mathcal{L}=\frac{1}{2} \rho \dot{w}^{2}-\frac{\kappa}{2}\left(\nabla^{2} w\right)^{2}-\frac{1}{2} \sigma_{i j} \epsilon_{i j}-\frac{1}{2} V(\mathbf{r}) w^{2} \\
(i, j=x, y)
\end{gathered}
$$

where $w(\mathbf{r})$ is the out-of-plane deformation, $\rho$ is the mass density, $\kappa$ is the bending rigidity, $\sigma_{i j}$ is the stress and $\epsilon_{i j}$ is the strain tensor $^{32}$. Here, we have used the out-of-plane approximation ${ }^{28}$, which is valid for large pre-strain typically found for suspended graphene sheets ${ }^{33}$. For isotropic pre-strain, $\epsilon_{i j} \rightarrow \varepsilon \delta_{i, j}+\partial_{i} w \partial_{j} w / 2$, the Lagrangian density can be further simplified. The pre-tension $T_{0}=\varepsilon(\lambda+2 \mu)$ determines the unit of frequency and $\lambda$ and $\mu$ are the Lamé parameters of the membrane. The pinning potential is given by $V(\mathbf{r})=V_{0} \sum_{n m} \Theta\left(\left|\mathbf{r}-\mathbf{r}_{n m}\right|-\sigma a\right)$, where $V_{0}$ is the pinning strength and $\mathbf{r}_{n m}=n \cdot \mathbf{a}_{1}+m \cdot \mathbf{a}_{2}$. Due to the periodicity of the pinning lattice, the system supports frequency bands analogous to the electronic bands in periodic solids. See Supplementary Fig. 1a for a calculation of the lowest lying frequency bands in the present system.

In the following we take the lattice spacing $a$ to be the unit of length, $a \sqrt{\rho / T_{0}}$ the unit of time and $\rho a^{2}$ the unit of mass.

Coupled localized modes. Considering two vacancies in the lattice, which are far apart, one finds two degenerate, localized modes. Moving the vacancies closer together, the degeneracy is lifted and one obtains a symmetric and an antisymmetric mode at frequencies $\Omega_{\mp}$. Figure 2a,b shows the mode shapes for $\sigma=1 / 5$. The frequency splitting indicates that the localized modes leak into the region of the neighbouring vacancy, which leads to an intermode interaction.

Figure $2 \mathrm{c}, \mathrm{d}$ shows the centre frequency $\left(\Omega_{+}+\Omega_{-}\right) / 2$ and the frequency difference $\Omega_{+}-\Omega_{-}$of a dimer for different pillar radii. The centre frequency increases with increasing $\sigma$, since the the size of the suspended region shrinks. The frequency difference on the other hand decreases, because the overlap of the localized modes vanishes. For an estimate of the scaling behaviour, one can consider the suspended region to be approximately a square of size $\xi \times \xi$. From a dimensional analysis one finds, that the frequency scales as $\Omega \propto 1 / \xi$. The length $\xi$ can be taken as a linear function of $\sigma$, as shown in Fig. 2c.

The interaction strength is calculated from the linearized equation of motion obtained from equation (1) by making the Ansatz $w(\mathbf{r})=\psi_{\mathrm{L}}(\mathbf{r}) q_{\mathrm{L}}+\psi_{\mathrm{R}}(\mathbf{r}) q_{\mathrm{R}}$. The localized mode shapes $\psi_{n}(\mathbf{r})$ are expressed by linear combinations of the exact dimer eigenmodes (Wannier basis) or estimated by using mode shapes of the single-vacancy modes (tight-binding approximation) ${ }^{34}$. In both cases, one obtains the generalized eigenvalue problem

$$
\Omega_{ \pm}^{2} \sum_{m} S_{m n} q_{m}=\sum_{m} V_{n m} q_{m}(m, n)=(\mathrm{L}, \mathrm{R})
$$

where the overlap matrix $S_{n m}=\left\langle\psi_{n} \mid \psi_{m}\right\rangle$, the interaction matrix $V_{n m}=\left\langle\nabla \psi_{n} \mid \nabla \psi_{m}\right\rangle+\left\langle\psi_{n} \mid V \psi_{m}\right\rangle$ and angular brackets denote spatial integration. The diagonal terms $\Omega_{n}^{2}=V_{n n}$ give the individual mode frequencies and the off-diagonal elements $\lambda_{n m}=V_{n m}$ are the linear coupling constants. In the tight-binding approximation, the overlap matrix $S_{n m}$ is not diagonal, but the values on the diagonal dominate. The effective interaction matrix is then obtained by multiplying $V_{n m}$ by $S_{n m}^{-1}$ from the left. From Fig. 2c,d, we find a good agreement between the tight binding and Wannier calculations for the chosen values of $\sigma$. This implies that an array of such defect modes support frequency bands which are well described by the tightbinding method (see Supplementary Fig. 1c and Supplementary Note 1). 

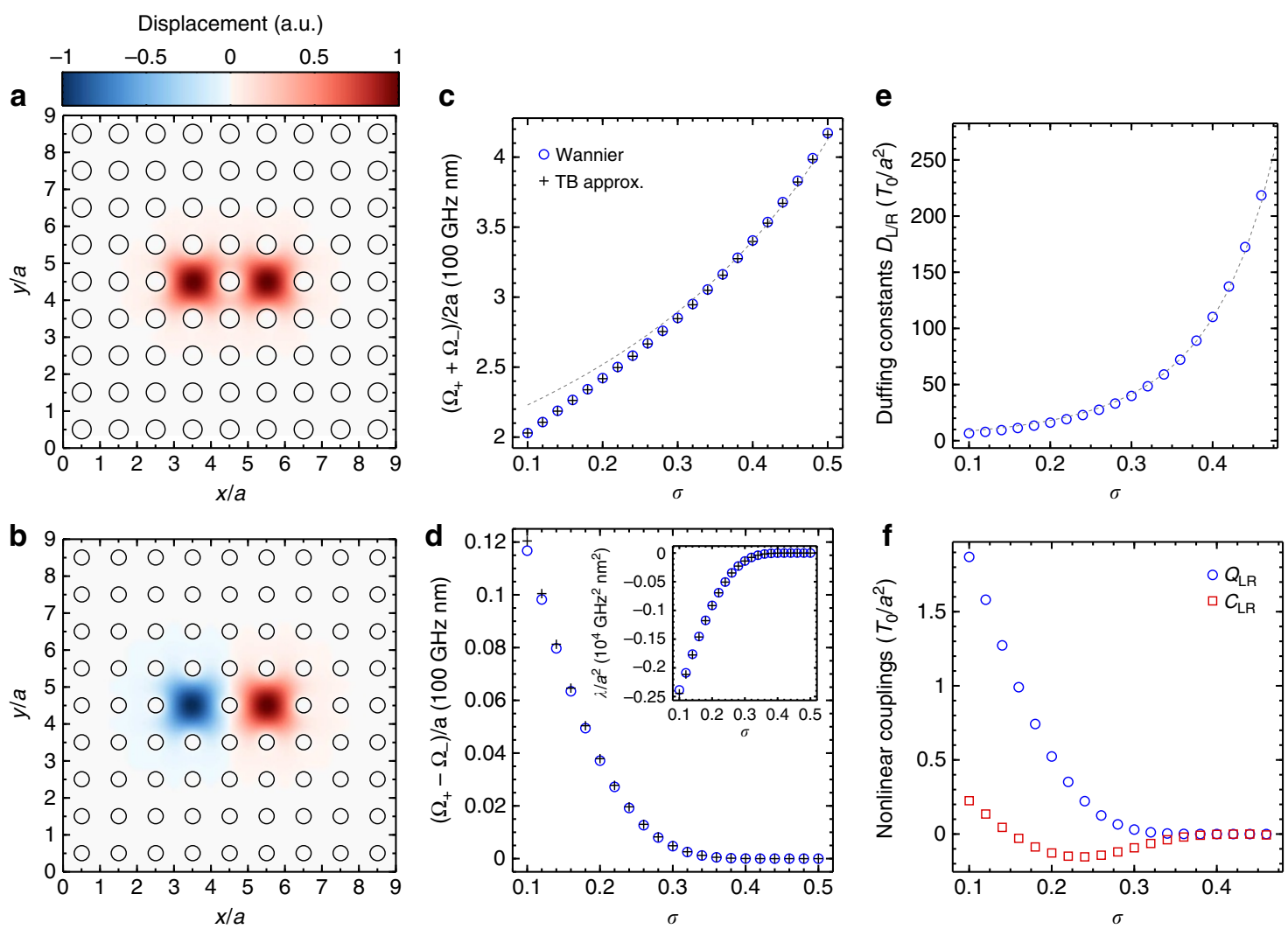

Figure 2 | Coupled localized modes. Displacement field of (a) symmetric and (b) antisymmetric modes in a lattice with two vacancies. The super cell contains $9 \times 9$ pillars (minus 2 for the vacancies) with radii $\sigma=1 / 5$. (c) Centre frequency and (d) frequency difference versus pillar radius $\sigma$. Inset shows the linear coupling strength versus pillar radius. Circles denote values obtained from the two lowest frequencies of the dimer, while plusses are estimates calculated from equation (2) in the tight-binding approximation. The dashed line in c corresponds to frequency $\Omega=2 /(1-1.03 \sigma)$, measured in units of $a^{-1} \sqrt{T_{0} / \rho}$ where $a$ is the lattice spacing, $\rho$ is the mass density of the membrane and $T_{0}$ is the pre-tension in the membrane. (e) Duffing constant, (f) quadratic and cubic coupling strength versus pillar radius $\sigma$. The dashed line in e shows Duffing constant $D_{n}=4.5 /(1-1.03 \sigma)^{6}$, in units of $T_{0} / a^{2}$.

Nonlinear effects. One consequence of the membrane being atomically thin is that geometrically induced nonlinear effects become appreciable already for moderate displacements. The respective contribution to the stretching energy in equation (1) is

$$
E_{\text {nlin }}=\frac{1}{8 \varepsilon}\left\langle\left[\left(\partial_{x} w\right)^{2}+\left(\partial_{y} w\right)^{2}\right]^{2}\right\rangle \text {. }
$$

This expression accounts for the energy required to accommodate for the area increase associated with a transverse deflection of the membrane.

To estimate the influence of the energy (equation (3)) for the dimer, we use the Wannier basis, that is, linear combinations of the eigenmodes for $w$. The energy is written as

$$
E_{\text {nlin }}=\frac{1}{8 \varepsilon}\left(D_{\mathrm{L}} q_{\mathrm{L}}^{4}+D_{\mathrm{R}} q_{\mathrm{R}}^{4}+Q_{\mathrm{LR}} q_{\mathrm{L}}^{2} q_{\mathrm{R}}^{2}+C_{\mathrm{LR}} q_{\mathrm{L}} q_{\mathrm{R}}^{3}+C_{\mathrm{RL}} q_{\mathrm{R}} q_{\mathrm{L}}^{3}\right),
$$

where $D_{\mathrm{L} / \mathrm{R}}, C_{\mathrm{LR} / \mathrm{RL}}$ and $Q_{\mathrm{LR}}$ are the Duffing, the cubic coupling and the the quadratic coupling constants, respectively (see Methods section).

Figure 2e,f shows the behaviour of $D_{\mathrm{LR}}, C_{\mathrm{LR}}$ and $Q_{\mathrm{LR}}$ as functions of $\sigma$. The Duffing constant increases with increasing $\sigma$ due to the shrinking suspension area. The nonlinear coupling constants, $C_{\mathrm{LR}}$ and $Q_{\mathrm{LR}}$, decrease analogous to the frequency splitting because the overlap of the localized modes vanishes. Using the same square-membrane approximation as for the centre frequency, one finds the scaling $D_{\mathrm{LR}} \propto 1 / \sigma^{6}$, which is in good agreement with the numerical data.
Dynamics in rotating wave approximation. Since the time scales associated with $\Omega \pm$ and the nonlinearities are well separated, it is useful to consider the dynamics of the coupled oscillator system in the rotating wave approximation (RWA). To this end, we define $q_{m}=\sqrt{1 / 2 \Omega_{0}}\left(a_{m} e^{-i \Omega_{0} t}+a_{m}^{*} e^{i \Omega_{0} t}\right)$ and $\dot{q}_{m}=-i \sqrt{\Omega_{0} / 2}\left(a_{m} e^{-i \Omega_{0} t}-a_{m}^{*} e^{i \Omega_{0} t}\right)$, where $\Omega_{0}$ is a reference frequency. The slowly changing amplitudes $a_{m}$ obey the equations of motion $\dot{a}_{m}=-i \partial H / \partial a_{m}^{*}$ (see also Supplementary Note 2$)^{35}$. For a symmetric system, that is, $D=D_{\mathrm{L} / \mathrm{R}}, C=C_{\mathrm{LR} / \mathrm{RL}}, \mathrm{Q}=\mathrm{Q}_{\mathrm{LR}}$ and $\lambda=\lambda_{\mathrm{LR} / \mathrm{RL}}$, the Hamiltonian $H$ in RWA is given by

$$
\begin{aligned}
H & \approx \frac{\delta \Omega_{\mathrm{L}}}{2}\left|a_{\mathrm{L}}\right|^{2}+\frac{\delta \Omega_{\mathrm{R}}}{2}\left|a_{\mathrm{R}}\right|^{2}+\frac{U}{2}\left(\left|a_{\mathrm{L}}\right|^{4}+\left|a_{\mathrm{R}}\right|^{4}\right) \\
& +\left[J+3 W\left(\left|a_{\mathrm{L}}\right|^{2}+\left|a_{\mathrm{R}}\right|^{2}\right)\right]\left[a_{\mathrm{L}}^{*} a_{\mathrm{R}}+a_{\mathrm{R}}^{*} a_{\mathrm{L}}\right] \\
& +K\left[\left|a_{\mathrm{R}}\right|^{2}\left|a_{\mathrm{L}}\right|^{2}+\frac{1}{4}\left(a_{\mathrm{L}}^{2}\left(a_{\mathrm{R}}^{*}\right)^{2}+a_{\mathrm{R}}^{2}\left(a_{\mathrm{L}}^{*}\right)^{2}\right)\right],
\end{aligned}
$$

where $\delta \Omega_{n}=\left(\Omega_{n}^{2}-\Omega_{0}^{2}\right) / \Omega_{0}$ is a small detuning from the reference frequency, and we have defined $J=\lambda / \Omega_{0}, \quad U=(3 D) /\left(8 \varepsilon \Omega_{0}^{2}\right)$, $K=Q /\left(8 \varepsilon \Omega_{0}^{2}\right)$ and $W=(3 C) /\left(32 \varepsilon \Omega_{0}^{2}\right)$ to characterize 'hopping', on-site interactions and interaction tunnelling, respectively. The values of $J, U, K$ and $W$ following from Fig. 2 are shown in Supplementary Fig. 2.

Equation (5) corresponds to the classical analogue of the improved two-mode model' 36 for Bose particles in a double-well potential. The Hamiltonian (equation (5)) can be expressed in terms of only two variables. Setting $a_{n}=\left|a_{n}\right| e^{i \phi_{n}}$, we define the 'population imbalance' $z=\left(\left|a_{\mathrm{R}}\right|^{2}-\left|a_{\mathrm{L}}\right|^{2}\right) / Z^{2}$ and the relative 
phase $\Delta \phi=\phi_{\mathrm{R}}-\phi_{\mathrm{L}}$. Note that the 'total population' $Z^{2}=\left|a_{\mathrm{R}}\right|^{2}+\left|a_{\mathrm{L}}\right|^{2}$ is conserved ${ }^{37}$. We further set the reference frequency to the average frequency, $\Omega_{0}^{2}=\left(\Omega_{\mathrm{L}}^{2}+\Omega_{\mathrm{R}}^{2}\right) / 2$, which maps equation (5) onto the Hamiltonian for a rigid rotor ${ }^{13,36}$

$$
\begin{aligned}
H_{\mathrm{eff}}= & \frac{U_{\mathrm{eff}} z^{2}}{2}+J_{\mathrm{eff}} \sqrt{1-z^{2}} \cos (\Delta \phi) \\
& +\frac{K_{\mathrm{eff}}}{2}\left(1-z^{2}\right) \cos (2 \Delta \phi)-\mu_{\mathrm{eff}} z,
\end{aligned}
$$

where $U_{\text {eff }}=(U-K) Z^{4} / 2, \quad J_{\text {eff }}=\left(J+W Z^{2}\right) Z^{2}, \quad K_{\text {eff }}=K Z^{4} / 4$ and $\mu_{\mathrm{eff}}=\left(\Omega_{\mathrm{L}}^{2}-\Omega_{\mathrm{R}}^{2}\right) Z^{2} / 4 \Omega_{0}$. The last term is due to detuning of the individual mode frequencies and tilts the double-well potential defined by this Hamiltonian. In the present system, $K_{\text {eff }} \ll \max \left(U_{\text {eff }}, J_{\text {eff }}\right)$ (see Supplementary Fig. 2), and the second to last term in equation (6) does not crucially influence the dynamics. Taking $K_{\mathrm{eff}} \rightarrow 0$, the corresponding equations of motion are

$$
\begin{gathered}
\dot{z}=J_{\text {eff }} \sqrt{1-z^{2}} \sin (\Delta \phi), \\
\Delta \dot{\phi}=U_{\text {eff }} z-J_{\text {eff }} \frac{z}{\sqrt{1-z^{2}}} \cos (\Delta \phi)-\mu_{\text {eff }} .
\end{gathered}
$$

For atoms in optical lattices, equation (6) is usually obtained in the semiclassical limit, for example by using the Gutzwiller Ansatz. Here, we derived the Hamiltonian (equation (6)) within a completely classical description employing the RWA (see also Supplementary Note 2). As a consequence, the total population $Z$ used above has the unit of an action and is proportional, but not equal to the total number of phonons.

For $\mu_{\text {eff }}=0$, the system undergoes a pitch-fork bifurcation at a critical value of the ratio $U_{\text {eff }} / J_{\text {eff }}$, with qualitatively different dynamics on each side of the bifurcation. This defines two regimes, denoted in the literature as the 'Rabi' regime for
$\mathrm{U}_{\text {eff }} / \mathrm{J}_{\text {eff }} \ll 1$ and 'Josephson' regime for $\mathrm{U}_{\text {eff }} / \mathrm{J}_{\text {eff }} \gg 1$ (ref. 15). The Rabi regime is characterized by oscillation of $z$ with vanishing temporal mean ${ }^{21}$. In the Josephson regime, there exist selftrapped fixed points at $z= \pm z_{0}$ and $\Delta \phi=0$, corresponding to either of the localized modes being predominantly excited. At even larger ratios $\left(U_{\text {eff }} / J_{\text {eff }}>2\right)$, running phase modes appear (Fig. 3b-d).

Note the different dependence of $U_{\text {eff }}$ and $J_{\text {eff }}$ on the vibrational amplitudes, entering through the parameter $Z$. This implies that the ratio can be tuned either through changes in the system itself (through the pillar radii), or by changing the vibrational amplitude of the dimer. In Fig. 3a, the ratio $U_{\text {eff }} / J_{\text {eff }}$ is shown as a function of the pillar radius and the vibrational amplitude scaled by the pre-strain. The dashed line corresponds to the transition between the two regimes. At a fixed pillar size, the system can be tuned into either regime through the vibrational amplitude.

Frequency tuning. External forces applied to the membrane will lead to a static deformation of the membrane. Due to the nonlinearities of the system, this can be used to tune the frequencies and couplings. Considering small oscillations around the static deformation, the equations of motion are linearized, which leads to the following renormalized frequencies and linear couplings

$$
\begin{gathered}
\Omega_{n}^{2} \rightarrow \Omega_{n}^{2}+\frac{3}{2 \varepsilon} \bar{q}_{n}^{2} D_{n}+\frac{1}{4 \varepsilon} Q_{n m} \bar{q}_{m}^{2}+\frac{3}{4 \varepsilon} C_{n m} \bar{q}_{m} \bar{q}_{n}, \\
\lambda_{n m} \rightarrow \lambda_{n m}+\frac{1}{2 \varepsilon} Q_{n m} \bar{q}_{n} \bar{q}_{m}+\frac{3}{8 \varepsilon} C_{n m}\left(\bar{q}_{m}^{2}+\bar{q}_{n}^{2}\right),
\end{gathered}
$$

where $\bar{q}_{n}$ are the static deflections. Assuming that the latter can be tuned independently for each oscillator, for instance via local back gates, this allows for complete control over the frequencies
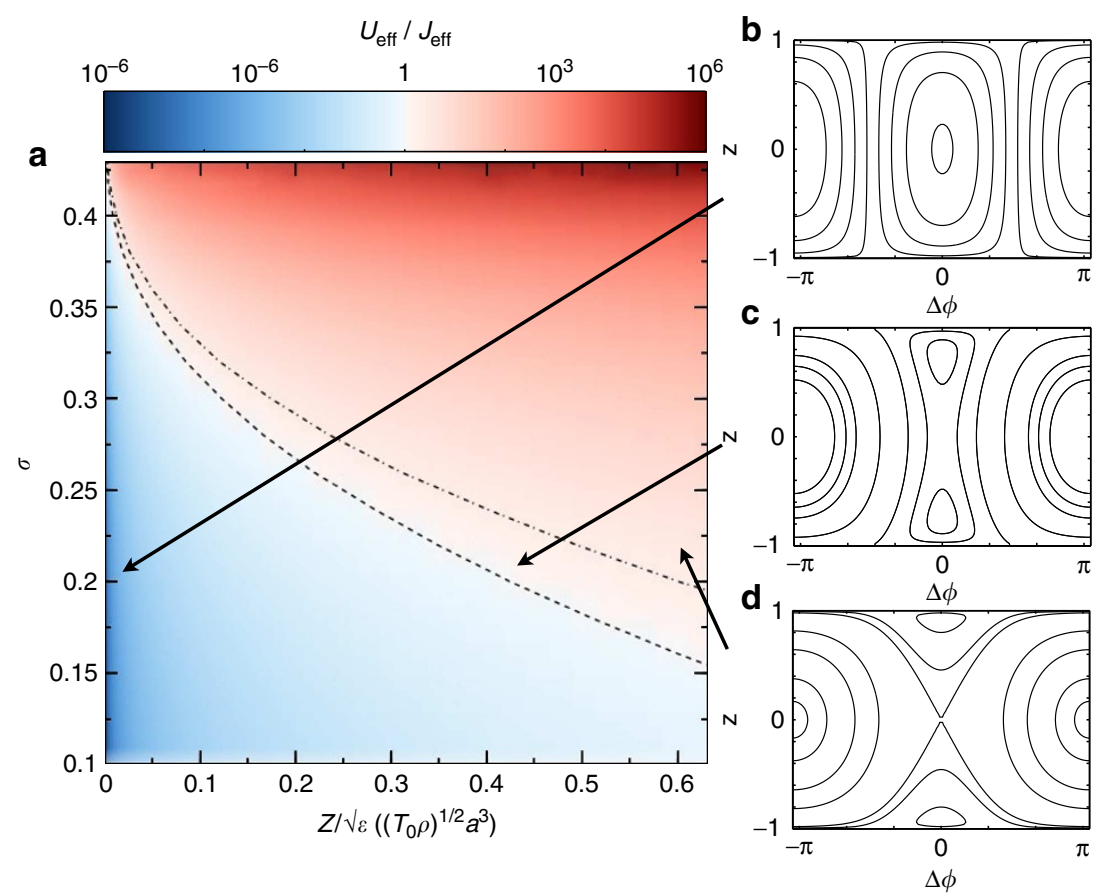

Figure 3 | Dynamical regimes of equation (6). (a) Ratio of nonlinear to linear interaction $U_{\text {eff }} / J_{\text {eff }}$ as a function of 'total population' $Z$ and pillar radius $\sigma$. By tuning the vibrational amplitude of the system, both the 'Rabi' (blue) and 'Josephson' (red) regimes are accessible The dashed line corresponds to $U_{\text {eff }} / J_{\text {eff }}=1$, while the dashed-dotted line corresponds to $U_{\text {eff }} / J_{\text {eff }}=2$. (b-d) Phase space plots of corresponding to the different regimes for tilt $\mu_{\text {eff }}=0$. (b) At low values of the ratio $\left(U_{\text {eff }} / J_{\text {eff }}=1 / 10\right)$, the system undergoes Rabi-like oscillations with vanishing temporal mean of the population imbalance $z$. (c): For values of the ratio $1<U_{\text {eff }} / J_{\text {eff }}<2$ (here $U_{\text {eff }} / J_{\text {eff }}=3 / 2$ ), the system develops two elliptic fixed points with $z$ having non vanishing temporal mean. (d) For higher ratios (here $U_{\text {eff }} / J_{\text {eff }}=3$ ) running phase modes appear. 


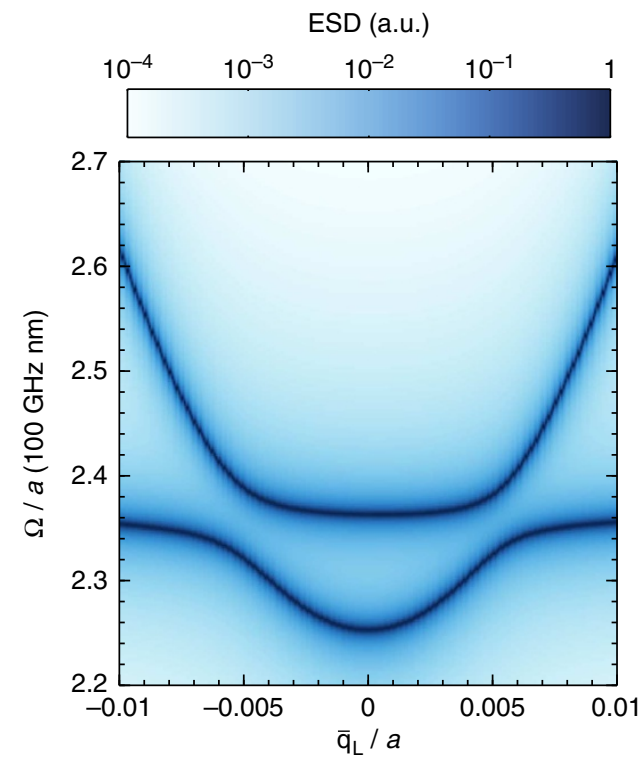

Figure 4 | Frequency tuning. Energy spectral density $(\operatorname{ESD}(\Omega))$ for the dimer as a function of static displacement $\bar{q}_{L}$ of the left mode and the driving frequency $\Omega$ for fixed static displacement $\bar{q}_{R}=0.005 a$, where $a$ is the lattice spacing. The data is scaled such that $\operatorname{ms}(\operatorname{ESD}(\Omega))=1$. Parameters are pillar radius $\sigma=1 / 5$, quality factor $Q \approx 500$ and pre-strain $\varepsilon=10^{-3}$

and the splitting. This effect is interesting for applications and may be used to study Landau-Zener transitions ${ }^{22}$ beyond the linear regime ${ }^{38}$.

To demonstrate the frequency tuning, we consider a harmonically driven dimer. The energy spectral density per unit driving amplitude $\operatorname{ESD}(\Omega)=\sum_{n} \Omega^{2}\left|\chi_{n}(\Omega)\right|^{2}$, where $\chi_{n}(\Omega)$ is the susceptibility for mode $n$, gives a measure of the response at the driving frequency $\Omega$. In Fig. 4 , the energy spectral density is shown as a function of the back gate induced static deflection. Here, $\bar{q}_{\mathrm{R}}$ is kept constant while $\bar{q}_{\mathrm{L}}$ is swept. Further, we assumed a quality factor of $Q \approx 500$ to account for damping of the mechanical motion.

\section{Discussion}

We have investigated the realization of a new type of phononic crystal, using periodically pinned and atomically thin membranes. Distorting the periodicity of the pinning lattice allows it to support coupled localized modes. Due to the geometric nonlinearity, the dynamics of the mode amplitudes is described by coupled nonlinear oscillator equations. By applying external forces, the nonlinearities allow tuning the frequencies and couplings.

Using typical values for graphene resonators $23,24,39,40$, $\lambda+2 \mu \approx 340 \mathrm{Nm}^{-1}, \quad \kappa \approx 1.5 \mathrm{eV}, \quad \rho \approx 7.6 \cdot 10^{-7} \mathrm{~kg} \mathrm{~m}^{-2}, \quad V_{0} \approx$ $1.8 \cdot 10^{20} \mathrm{Nm}^{-3}$ and $\varepsilon=0.1 \%$, one finds for the unit of frequency $(100 \mathrm{GHz} \mathrm{nm}) / a$ and the dimensionless pinning strength $a^{2} V_{0} / T_{0}=\left(500 \mathrm{~nm}^{-2}\right) a^{2}$. Assuming a lattice spacing of $100 \mathrm{~nm}$ therefore leads to frequencies in the $\mathrm{GHz}$ range. The graphene sheet can be treated as a membrane, if the bending energy in equation (1) can be neglected. In our case, this contribution becomes negligible for $a \gg \sqrt{\kappa / \varepsilon(\lambda+2 \mu)}$, which corresponds to $a \gg 8 \AA$. Then, the only parameters entering the problem are the details of the pinning potential, more specifically the pinning strength and the pillar radius. In the limit of strong pinning, the membrane will essentially be clamped at the pillars $(w=0)$. Recently, structures with graphene sheets suspended between silica pillars have been experimentally realized ${ }^{41}$.
A natural extension of the dimer configuration considered in the Results is a periodic array of defect modes. Analogous to the dimer, the energy of such a system can be expressed in terms of the amplitudes of localized (Wannier) modes. In general, the resulting Hamiltonian in RWA is given by

$$
H=\sum_{n}\left(\frac{\delta \Omega_{n}}{2}\left|a_{n}\right|^{2}+\sum_{m \neq n} \frac{J_{m n}}{2}\left[a_{m}^{*} a_{n}+a_{n}^{*} a_{m}\right]+\sum_{m, i, j} W_{n m, i j} a_{n}^{*} a_{m}^{*} a_{i} a_{j}\right),
$$

where $J_{n m}$ and $W_{n m, i j}$ denote 'hopping' and interaction constants, respectively. For a square lattice with dominating nearest neighbour couplings, the interaction energy can be constructed from the dimer contributions (see Supplementary Note 1). In that case, the hopping is restricted to nearest neighbours and the interaction term only contains contributions from at most two neighbouring modes. More general types of interactions can be realized by using other lattice configurations. The equations of motion for the mode amplitudes ensuing from equation (9) are the classical version of a discrete nonlinear Schrödinger equation, known to display rich nonlinear phenomena ${ }^{37,42}$.

To actuate the motion of the oscillators, external pressure may be applied to the membrane. In nanoelectromechanical systems, this is typically achieved by the electrostatic interaction with a metallic back gate ${ }^{25}$. This leads to the pressure $P_{z} \propto-G(\mathbf{r}) V_{\mathrm{g}}^{2}$ with $V_{\mathrm{g}}$ denoting the gate voltage and $G(\mathbf{r})$ the shape of the gate ${ }^{28}$. The force acting on each oscillator is given by $f_{n}^{(\text {e } x t)} \propto-\left\langle\psi_{n} \mid G\right\rangle V_{\mathrm{g}}^{2}$. A d.c. voltage leads to a static displacement $\bar{q}_{n}>0$ in equation (8), while an a.c. voltage can be used to drive the system.

The coupling of the membrane to its environment leads to dissipation of the mechanical motion. This has been extensively studied in the context of atomically thin membrane resonators ${ }^{43}$, and quality factors of $Q>100,000$ have been reported for graphene resonators at low temperatures ${ }^{26,27}$. Phenomenologically, the dissipation can be described by a viscous damping term proportional to $Q^{-1} \dot{q}_{n}$ in the equations of motion. In the Rabi regime, the oscillation frequency is given by $|J|$ and $Q \gg 1 /$ $|J|$ is required. We find for $\sigma=1 / 5$, that $1 /|J| \approx 65$ and $Q \approx 500$ would be sufficient to resolve the oscillations. In addition to the viscous damping, nonlinear dissipation has been observed in carbon based resonators ${ }^{26}$, which might give rise to interesting nonlinear dissipation effects ${ }^{17}$.

Due to the high frequencies of the localized modes and the low mass of the membrane, our system is a promising candidate for studies in the quantum regime ${ }^{29}$. The presence of intrinsic nonlinearities makes it attractive for creation and detection of non-classical states ${ }^{44-46}$. More generally, realizing lattices of interacting flexural modes would pave the road for quantum many-body phononics.

\section{Methods}

Nonlinear constants. Using the Ansatz $w(\mathbf{r})=\psi_{\mathrm{L}}(\mathbf{r}) q_{\mathrm{L}}+\psi_{\mathrm{R}}(\mathbf{r}) q_{\mathrm{R}}$ in equation (3) leads to the expression of the energy (equation (4)) in terms of the nonlinear constants

$$
\begin{gathered}
D_{n}=\left\langle\left[\left(\partial_{x} \psi_{n}\right)^{2}+\left(\partial_{x} \psi_{n}\right)^{2}\right]^{2}\right\rangle \\
C_{\mathrm{RL}}=4\left\langle\left[\left(\partial_{x} \psi_{\mathrm{L}}\right)^{2}+\left(\partial_{y} \psi_{\mathrm{L}}\right)^{2}\right] \times\left[\left(\partial_{x} \psi_{\mathrm{L}}\right)\left(\partial_{x} \psi_{\mathrm{R}}\right)+\left(\partial_{y} \psi_{\mathrm{L}}\right)\left(\partial_{y} \psi_{\mathrm{R}}\right)\right]\right\rangle \\
C_{\mathrm{LR}}=4\left\langle\left[\left(\partial_{x} \psi_{\mathrm{R}}\right)^{2}+\left(\partial_{y} \psi_{\mathrm{R}}\right)^{2}\right] \times\left[\left(\partial_{x} \psi_{\mathrm{R}}\right)\left(\partial_{x} \psi_{\mathrm{L}}\right)+\left(\partial_{y} \psi_{\mathrm{R}}\right)\left(\partial_{y} \psi_{\mathrm{L}}\right)\right]\right\rangle \\
\mathrm{Q}_{\mathrm{LR}}=2\left\langle 4\left(\partial_{x} \psi_{\mathrm{L}}\right)\left(\partial_{x} \psi_{\mathrm{R}}\right)\left(\partial_{y} \psi_{\mathrm{L}}\right)\left(\partial_{y} \psi_{\mathrm{R}}\right)\right. \\
+\left(\partial_{x} \psi_{\mathrm{L}}\right)^{2}\left[\left(\partial_{y} \psi_{\mathrm{R}}\right)^{2}+3\left(\partial_{x} \psi_{\mathrm{R}}\right)^{2}\right] \\
\left.+\left(\partial_{x} \psi_{\mathrm{L}}\right)^{2}\left[\left(\partial_{x} \psi_{\mathrm{R}}\right)^{2}+3\left(\partial_{y} \psi_{\mathrm{R}}\right)^{2}\right]\right\rangle
\end{gathered}
$$




\section{References}

1. Soljacic', M. \& Joannopoulos, J. Enhancement of nonlinear effects using photonic crystals. Nat. Mater. 3, 211-219 (2004).

2. Eichenfield, M., Chan, J., Camacho, R. M., Vahala, K. J. \& Painter, O. Optomechanical crystals. Nature 462, 78-82 (2009).

3. Heinrich, G., Ludwig, M., Qian, J., Kubala, B. \& Marquardt, F. Collective dynamics in optomechanical arrays. Phys. Rev. Lett. 107, 043603 (2011).

4. Ludwig, M. \& Marquardt, F. Quantum many-body dynamics in optomechanical arrays. Phys. Rev. Lett. 111, 073603 (2013).

5. Jaksch, D., Bruder, C., Cirac, J. I., Gardiner, C. W. \& Zoller, P. Cold bosonic atoms in optical lattices. Phys. Rev. Lett. 81, 3108-3111 (1998).

6. Bloch, I. Ultracold quantum gases in optical lattices. Nat. Phys. 1, 23-30 (2005).

7. Morsch, O. \& Oberthaler, M. Dynamics of Bose-Einstein condensates in optical lattices. Rev. Mod. Phys. 78, 179-215 (2006).

8. Berman, G. P. \& Izrailev, F. M. The Fermi-Pasta-Ulam problem: fifty years of progress. Chaos 15, 015104 (2005).

9. Lu, M.-H., Feng, L. \& Chen, Y.-F. Phononic crystals and acoustic metamaterials. Mater. Today 12, 34-42 (2009).

10. Balandin, A. A. \& Nika, D. L. Phononics in low-dimensional materials. Mater. Today 15, 266-275 (2012).

11. Maldovan, M. Sound and heat revolutions in phononics. Nature 503, 209-217 (2013).

12. Boechler, N., Theocharis, G. \& Daraio, C. Bifurcation-based acoustic switching and rectification. Nat. Mater. 10, 665-668 (2011).

13. Smerzi, A., Fantoni, S., Giovanazzi, S. \& Shenoy, S. R. Quantum coherent atomic tunneling between two trapped Bose-Einstein condensates. Phys. Rev. Lett. 79, 4950-4953 (1997).

14. Albiez, M. et al. Direct observation of tunneling and nonlinear self-trapping in a single bosonic Josephson junction. Phys. Rev. Lett. 95, 010402 (2005).

15. Zibold, T., Nicklas, E., Gross, C. \& Oberthaler, M. K. Classical bifurcation at the transition from Rabi to Josephson dynamics. Phys. Rev. Lett. 105, 204101 (2010).

16. Shim, S.-B., Imboden, M. \& Mohanty, P. Synchronized oscillation in coupled nanomechanical oscillators. Science 316, 95-99 (2007).

17. Lifshitz, R. \& Cross, M. Nonlinear Dynamics of Nanomechanical and Micromechanical Resonators (Wiley-VCH, 2008).

18. Karabalin, R., Cross, M. \& Roukes, M. Nonlinear dynamics and chaos in two coupled nanomechanical resonators. Phys. Rev. B 79, 165309 (2009).

19. Tsioutsios, I., Moser, J., Plaza, J. A. \& Bachtold, A. Controlled assembly of graphene sheets and nanotubes: Fabrication of suspended multi-element allcarbon vibrational structures. J. Appl. Phys. 114, 104310 (2013).

20. Okamoto, H. et al. Coherent phonon manipulation in coupled mechanical resonators. Nat. Phys. 9, 480-484 (2013).

21. Faust, T., Rieger, J., Seitner, M. J., Kotthaus, J. P. \& Weig, E. M. Coherent control of a classical nanomechanical two-level system. Nat. Phys. 9, 485-488 (2013).

22. Faust, T. et al. Nonadiabatic dynamics of two strongly coupled nanomechanical resonator modes. Phys. Rev. Lett. 109, 037205 (2012).

23. Lee, C., Wei, X., Kysar, J. W. \& Hone, J. Measurement of the elastic properties and intrinsic strength of monolayer graphene. Science 321, 385-388 (2008).

24. Lindahl, N. et al. Determination of the bending rigidity of graphene via electrostatic actuation of buckled membranes. Nano Lett. 12, 3526-3531 (2012).

25. Song, X. et al. Stamp transferred suspended graphene mechanical resonators for radio frequency electrical readout. Nano Lett. 12, 198-202 (2012).

26. Eichler, A. et al. Nonlinear damping in mechanical resonators made from carbon nanotubes and graphene. Nat. Nanotechnol. 6, 339-342 (2011).

27. Weber, P., Güttinger, J., Tsioutsios, I., Chang, D. E. \& Bachtold, A. Coupling graphene mechanical resonators to superconducting microwave cavities. Nano Lett. 14, 2854-2860 (2014).

28. Atalaya, J., Isacsson, A. \& Kinaret, J. M. Continuum elastic modeling of graphene resonators. Nano Lett. 8, 4196-4200 (2008).
29. Poot, M. \& van der Zant, H. S. J. Mechanical systems in the quantum regime Phys. Rep. 511, 273-335 (2012).

30. Viola Kusminskiy, S., Campbell, D. K., Castro Neto, A. H. \& Guinea, F. Pinning of a two-dimensional membrane on top of a patterned substrate: the case of graphene. Phys. Rev. B 83, 165405 (2011).

31. Medvedyeva, M. V. \& Blanter, Y. M. Eigenfrequencies of the randomly pinned drum and conductivity of graphene. Phys. Rev. B 88, 125423 (2013).

32. Landau, L. D. \& Lifshitz, E. M. Theory of Elasticity 3rd edn (ButterworthHeinemann, 1986).

33. Roldán, R., Fasolino, A., Zakharchenko, K. V. \& Katsnelson, M. I. Suppression of anharmonicities in crystalline membranes by external strain. Phys. Rev. B 83, 174104 (2011).

34. Ashcroft, N. W. \& Mermin, N. D. Solid State Physics (Saunders College, 1976).

35. Briggs, J. S. \& Eisfeld, A. Coherent quantum states from classical oscillator amplitudes. Phys. Rev. A 85, 052111 (2012).

36. Ananikian, D. \& Bergeman, T. Gross-Pitaevskii equation for bose particles in a double-well potential: two-mode models and beyond. Phys. Rev. A 73, 013604 (2006).

37. Eilbeck, J. C. \& Johansson, M. in Localization and Energy Transfer in Nonlinear Systems. (eds Vazquez, L., MacKay, R. S. \& Zorzano, M. P.) (World Scientific Publishing, 2003)

38. Wu, B. \& Niu, Q. Nonlinear Landau-Zener tunneling. Phys. Rev. A 61, 023402 (2000).

39. Persson, B. N. J. \& Ueba, H. Heat transfer between weakly coupled systems: graphene on a-SiO 2 . Europhys. Lett. 91, 56001 (2010).

40. Kudin, K. N., Scuseria, G. E. \& Yakobson, B. I. $C_{2} F, B N$, and $C$ nanoshell elasticity from $a b$ initio computations. Phys. Rev. B 64, 235406 (2001).

41. Reserbat-Plantey, A. et al.Strain superlattices and macroscale suspension of graphene induced by corrugated substrates. Preprint at http://arxiv.org/abs/ 1404.5783.

42. Eilbeck, J. C., Lomdahl, P. S. \& Scott, A. C. The discrete self-trapping equation Phys. D 16, 318-338 (1985).

43. Imboden, M. \& Mohanty, P. Dissipation in nanoelectromechanical systems. Phys. Rep. 534, 89-146 (2014).

44. Voje, A., Kinaret, J. M. \& Isacsson, A. Generating macroscopic superposition states in nanomechanical graphene resonators. Phys. Rev. B 85, 205415 (2012).

45. Voje, A., Croy, A. \& Isacsson, A. Multi-phonon relaxation and generation of quantum states in a nonlinear mechanical oscillator. New J. Phys. 15, 053041 (2013).

46. Voje, A., Isacsson, A. \& Croy, A. Nonlinear-dissipation-induced entanglement of coupled nonlinear oscillators. Phys. Rev. A 88, 022309 (2013).

\section{Acknowledgements}

We acknowledge funding from the Swedish Research Council and from the European Union through project no. 604391 (Graphene Flagship).

\section{Author contributions}

All authors contributed equally to the work presented in this paper.

\section{Additional information}

Supplementary Information accompanies this paper at http://www.nature.com/ naturecommunications

Competing financial interests: The authors declare no competing financial interests.

Reprints and permission information is available online at http://npg.nature.com/ reprintsandpermissions/

How to cite this article: Midtvedt, D. et al. Nonlinear phononics using atomically thin membranes. Nat. Commun. 5:4838 doi: 10.1038/ncomms5838 (2014). 